\title{
I atrogenias: ações do enfermeiro na prevenção de ocorrências iatrogênicas em unidade de terapia intensiva
}

\author{
Iatrogenic: actions of nurses in the prevention of iatrogenic occurrences in the \\ intensive care unit
}

\author{
Iatrogenias: las acciones de los enfermeros en la prevención de ocurrencias \\ iatrogénicas en la unidad de cuidados intensivos
}

\begin{abstract}
Resumo: A enfermagem é uma profissão que vem se desenvolvendo pelos séculos tendo como essência o cuidado ao ser humano, pois além de ciência, a enfermagem é uma profissão com um papel espetacular. O trabalho em unidade de terapia intensiva (UTI) é complexo e intenso, devendo o enfermeiro estar preparado para a qualquer momento. O objetivo deste estudo foi identificar as principais iatrogenias de enfermagem na UTI e o papel do enfermeiro na prevenção dessas ocorrências na unidade de terapia intensiva. Estudo de revisão bibliográfica acerca da iatrogenia e quais as ações do enfermeiro na prevenção dessas iatrogenias com amostra composta por 20 artigos consultados à base de dados da Biblioteca Virtual de Saúde e Biblioteca Física, artigos publicados no período de 2000 a 2011, utilizando os seguintes descritores: iatrogenias, enfermagem, erros de medicação, unidade de terapia intensiva e educação em saúde. Concluímos que o processo de prevenção das ocorrências iatrogênicas na UTI requer esforço contínuo de todos dos membros da equipe que atuam no setor e não apenas do enfermeiro.
\end{abstract}

Descritores: Iatrogenia, Enfermagem, Unidade de Terapia Intensiva.

\begin{abstract}
Nursing is a profession that has developed over the centuries as the essence with the care for human beings, as well as science, nursing is a profession with an outstanding role. The work in the intensive care unit (ICU) is complex and intense, the nurse should be prepared at any moment. The objective of this study was to identify the main iatrogenic in ICU nursing and nurse's role in preventing such occurrences in the intensive care unit. Bibliographic review about the iatrogenic and what actions the nurse in the prevention of iatrogenic complications with sample consisting of 20 articles referred to the data base of the Library Virtual Health and Library Physics papers published in the period 2000 to 2011, using the following descriptors: iatrogenic, nursing, medication errors, ICU and health education. We conclude that the process of prevention of iatrogenic occurrences in the ICU requires continuous effort of all team members who work in the industry and not just the nurse.
\end{abstract}

Descriptors: Iatrogenic, Nursing < Intensive Care Unit.

Resumen: Nursing is a profession that has developed over the centuries as the essence with the care for human beings, as well as science, nursing is a profession with an outstanding role. The work in the intensive care unit (ICU) is complex and intense, the nurse should be prepared at any moment. The objective of this study was to identify the main iatrogenic in ICU nursing and nurse's role in preventing such occurrences in the intensive care unit. Bibliographic review about the iatrogenic and what actions the nurse in the prevention of iatrogenic complications with sample consisting of 20 articles referred to the data base of the Library Virtual Health and Library Physics papers published in the period 2000 to 2011, using the following descriptors: iatrogenic, nursing, medication errors, ICU and health education. We conclude that the process of prevention of iatrogenic occurrences in the ICU requires continuous effort of all team members who work in the industry and not just the nurse.

Descriptores: Iatrogenia, Enfermería, Unidad de Cuidados Intensivos.

Luiz Faustino dos Santos Maia Enfermeiro. Mestre em Terapia Intensiva pela SOBRATI. Especialista em Saúde Coletiva e Saúde da Família; Gestão e Auditoria dos Serviços de Enfermagem; Enfermagem em Urgência, Emergências e Cuidados Intensivos pela UNICSUL; Programa Especial de Formação Pedagógica em Ciências Biológicas pela UNINOVE; Docência do Ensino Médio, Técnico e Superior na Área da Saúde pela FAPI. Docente de graduação em Enfermagem pela FMS e FALC. Coordenador Geral da Revista Recien. E-mail: dr.luizmaia@yahoo.com.br 
latrogenias: ações do enfermeiro na prevenção de ocorrências iatrogênicas em unidade de terapia intensiva

\section{I ntrodução}

A enfermagem como ciência vem se desenvolvendo ao longo das últimas décadas e uma estreita relação com a história da humanidade ${ }^{1}$, a começar desde os primórdios com Florence Nightingale (1820 - 1910) foi uma enfermeira britânica que ficou famosa por ser pioneira no tratamento a feridos de guerra.

A enfermagem é uma profissão que vem se desenvolvendo pelos séculos tendo como essência o cuidado ao ser humano, sendo responsável pela promoção, prevenção e a recuperação da saúde, individual, familiar e em comunidade. Nesse contexto, promove o bem estar e preza pela integridade física e mental das pessoas, respeitando suas diferenças e afirmando a unicidade de cada ser, buscando a igualdade, liberdade e dignidade para as pessoas ${ }^{2}$.

Além de ciência, a enfermagem é uma profissão com um papel espetacular, na medida em que busca a promoção do bem estar do ser humano e uma melhor qualidade de vida para a comunidade ${ }^{1}$.

O trabalho em unidade de terapia intensiva (UTI) é complexo e intenso, devendo o enfermeiro estar preparado para a qualquer momento, atender pacientes com alterações hemodinâmicas importantes, as quais requerem conhecimento específico e grande habilidade para tomar decisões e implementá-las em tempo hábil. Desta forma, pode-se supor que o enfermeiro desempenha importante papel no âmbito da $\mathrm{UTI}^{3}$.

O cuidado intensivo dispensado a pacientes críticos tornam-se mais eficaz quando desenvolvido em unidades específicas, que propiciam recursos e facilidades para a sua progressiva recuperação ${ }^{4}$.

O presente estudo busca trazer uma revisão da literatura acerca da iatrogenia e quais as ações do enfermeiro na prevenção dessas iatrogenias no contexto da unidade de terapia intensiva.

Os primeiros registros sobre latrogenia datam de civilizações antigas, e são definidas como eventos indesejáveis, não planejados, que causam ou tem o potencial de ocasionar resultados prejudiciais ao estado físico ou mental do paciente, podendo ou não ser resultado de negligência ou falha do profissional envolvido com a assistência ${ }^{5}$.
O termo iatrogenia é uma palavra de origem grega que define o resultado indesejável pela ação prejudicial não intencional dos profissionais de saúde. Relacionado à observação, monitorização ou intervenção terapêutica, caracterizando uma falha profissional por negligência e percebemos quão pouco tem se discutido por melhores condições de recuperação da saúde para os pacientes internados nos hospitais ${ }^{6}$.

\section{Justificativa}

A decisão por este tema tem o intuito de elucidar como podem ocorrer as iatrogenias, dando ênfase a sua prevenção. Inclui-se como meta a ação educativa e preventiva para que se possa ter melhor qualidade na assistência dirigida ao paciente.

Conscientizar a equipe de multiprofissional da importância na prevenção das iatrogenias, pois podem prolongar a hospitalização, dificultando a recuperação do paciente e aumentando o risco para o desenvolvimento de outras complicações. É por representarem um acréscimo no sofrimento físico e emocional dos pacientes, reduzindo a sua independência e funcionalidade na realização das atividades da vida diária, comprometendo qualquer processo reeducacional, que as iatrogenias merecem por parte da equipe multiprofissional toda atenção, no sentido de prevenir o seu aparecimento ou favorecer o seu tratamento.

Portando, é necessário que não só os enfermeiros, mas toda equipe multiprofissional sinta-se envolvida e comprometida em conhecer e entender o que são as iatrogenias, suas causas e os fatores de riscos, a fim de implementar ações efetivas e tratamento.

\section{Objetivo}

O objetivo deste estudo foi identificar as principais iatrogenias de enfermagem na UTI e o papel do enfermeiro na prevenção dessas ocorrências na unidade de terapia intensiva.

- Explorar o conceito de iatrogenia;

- Fazer um levantamento dos artigos e publicações que versam sobre o tema;

- Descrever a contribuição do enfermeiro na prevenção de iatrogenias. 
latrogenias: ações do enfermeiro na prevenção de ocorrências iatrogênicas em unidade de terapia intensiva

\section{Material e Método}

A complexidade das questões suscitadas levou-nos a optar por uma pesquisa exploratória bibliográfica, pois esta pesquisa proporciona maior familiaridade com o problema levantado, com vistas a torná-lo mais explicito ou a construir hipóteses mais sustentáveis. Apresenta ainda a vantagem de permitir ao investigador a cobertura de fenômenos de forma ampliada, o que de certa maneira seria impraticável em uma investigação direta ${ }^{7}$.

Após a escolha do tema, realizamos um levantamento bibliográfico preliminar a fim de justificar sua escolha.

\section{Procedimentos de Coleta de Dados}

O delineamento da pesquisa foi orientado pelos seguintes critérios:

a. Bases de Dados de Pesquisa: Biblioteca Virtual de Saúde, revistas e biblioteca física;

b. Descritores: iatrogenias, enfermagem, erros de medicação, unidade de terapia intensiva e educação em saúde;

c. Tipo de publicações: somente artigos científicos;

d. Idiomas das publicações: somente artigos publicados em língua portuguesa;

e. Período de tempo: artigos publicados entre 2000 e 2011.

A seleção do material se processará através da leitura sistemática dos resumos online, sendo esta orientada pelos seguintes critérios:

a. O artigo deve discorrer sobre ações do enfermeiro para prevenção de iatrogenias em unidades de terapia intensiva.

b. Os artigos devem atender a todos os critérios de delineamento.

Após a seleção dos trabalhos será realizada a obtenção dos textos e leitura na íntegra dos mesmos.

O material obtido foi classificado quantitativamente conforme o Instrumento de Coleta de Dados (ANEXO I), a seguir, analisado e apresentado por meio de tabelas com distribuição de percentual.

\section{Resultados e Discussão}

\section{Caracterização da Literatura Selecionada}

Procederemos à caracterização da literatura selecionada para evidenciar o rigor metodológico.
Para compor o conhecimento sobre o tema pesquisado, analisamos 20 artigos que atenderam os critérios de inclusão estabelecidos previamente, montando-se assim uma tabela com as conclusões sobre o conteúdo destes artigos. A tabela 1 descreve a distribuição das publicações conforme os descritores e base de dados.

Tabela 1. Distribuição de publicações segundo descritores e Bases de Dados. São Paulo, 2012

\begin{tabular}{|c|c|c|c|c|}
\hline \multirow{3}{*}{ Descritores } & \multicolumn{4}{|c|}{ Bases de Dados } \\
\hline & \multicolumn{2}{|c|}{$\begin{array}{l}\text { Biblioteca } \\
\text { Virtual }\end{array}$} & \multicolumn{2}{|c|}{$\begin{array}{l}\text { Biblioteca } \\
\text { Física }\end{array}$} \\
\hline & E & $\mathbf{S}$ & $E$ & S \\
\hline latrogenias, Enfermagem & & Refino & & Refino \\
\hline Iatrogenias + Enfermagem & 38 & 08 & 03 & 01 \\
\hline Iatrogenias + UTI & 27 & 05 & 06 & 01 \\
\hline Enfermagem + Erros de Medicação & 09 & 02 & 02 & -- \\
\hline Enfermagem + UTI & 11 & 01 & 02 & --- \\
\hline Enfermagem + Educação em Saúde & 03 & 01 & 04 & 01 \\
\hline Total de Artigos Encontrados & 88 & & 17 & \\
\hline Total de Artigos Selecionados & & 17 & & 03 \\
\hline
\end{tabular}

Fonte: Biblioteca Virtual e Física, 2011.

Observação: $\mathrm{E}=$ encontrados; $\mathrm{S}=$ selecionados.

A tabela acima evidencia como foram realizados os cruzamentos dos descritores. A seleção dos artigos seguiu o plano de trabalho descrito no capítulo metodologia deste estudo.

Tabela 2. Distribuição de artigos segundo período (ano) de publicação. São Paulo, 2012

\begin{tabular}{|c|c|c|}
\hline Período de Publicação & $\mathrm{N}^{\circ}$ de Artigos & $\%$ \\
\hline $2000 \mid-2003$ & 07 & 35,0 \\
\hline $2004 \mid 2007$ & 04 & 20,0 \\
\hline $2008 \mid-2011$ & 09 & 45,0 \\
\hline Total & 20 & $100 \%$ \\
\hline
\end{tabular}

A tabela 2 evidencia que houve uma constância em publicações a partir de 2000, houve maior incidência entre 2008 a 2011 com 45,0\% das publicações pesquisadas, no entanto, o número de artigos publicados não tem expressividade numérica.

Tabela 3. Distribuição de artigos selecionados segundo autoria.

\begin{tabular}{lcl}
\multicolumn{1}{c}{ Autoria } & $\mathbf{N}^{\circ}$ de Artigos & \% \\
\hline \hline Docentes & 06 & 30,0 \\
Docentes e Discentes & 14 & 70,0 \\
\hline Total & $\mathbf{2 0}$ & $\mathbf{1 0 0 \%}$ \\
\hline \hline
\end{tabular}

Fonte: Biblioteca Virtual e Física, 2011.

$\mathrm{Na}$ tabela 3 quanto à autoria dos artigos publicados podemos observar que a maioria $(70,0 \%)$ tem a participação de docentes e discentes na pesquisa. Este fato nos leva a questionar a necessidade de publicação com enfermeiros de campo ou assistência direta. 
latrogenias: ações do enfermeiro na prevenção de ocorrências iatrogênicas em unidade de terapia intensiva

Tabela 4. Distribuição de artigos selecionados segundo indexação. São Paulo, 2012.

\begin{tabular}{ccc}
\multicolumn{3}{c}{ indexação. São Paulo, 2012. } \\
\hline \hline Indexação & $\mathbf{N}^{\circ}$ de Artigos & $\%$ \\
\hline $\mathrm{A}_{2}$ & 09 & 45,0 \\
$\mathrm{~B}_{1}$ & 02 & 10,0 \\
$\mathrm{~B}_{3}$ & 01 & 5,0 \\
$\mathrm{~B}_{4}$ & 01 & 5,0 \\
$\mathrm{~B}_{5}$ & 01 & 5,0 \\
Não Indexada & 06 & 30,0 \\
\hline Total & $\mathbf{2 0}$ & $\mathbf{1 0 0 \%}$ \\
\hline \hline
\end{tabular}

Tabela 5. Descrição da amostra da pesquisa. São Paulo, 2012

\begin{tabular}{|c|c|c|}
\hline Autor & Título & Ano \\
\hline Maia LFS, Alves FG. & $\begin{array}{l}\text { O papel do enfermeiro na prevenção de ocorrências iatrogênicas em unidade } \\
\text { de terapia intensiva. }\end{array}$ & 2011 \\
\hline $\begin{array}{l}\text { Farias GM, Costa IKF, Rocha } \\
\text { KMM, Fritas MCS, Dantas } \\
\text { RAN. }\end{array}$ & $\begin{array}{l}\text { latrogenias na assistência de enfermagem: características da produção } \\
\text { científica no período de } 2000 \text { a } 2009 \text {. }\end{array}$ & 2010 \\
\hline $\begin{array}{l}\text { Ribeiro MPFN, Teodoro MWR, } \\
\text { Borges OS, Brasileiro ME. }\end{array}$ & $\begin{array}{l}\text { Ocorrências iatrogênicas nas unidades de terapia intensiva: enfoque nas ações } \\
\text { da equipe de enfermagem. }\end{array}$ & 2010 \\
\hline Vargas MAO, Ramos FRS. & $\begin{array}{l}\text { latrogenias nas unidades de terapia intensiva: dramaticidade dos problemas } \\
\text { bio/éticos contemporâneos. }\end{array}$ & 2010 \\
\hline $\begin{array}{l}\text { Cortez EA, Marçal C, Cardoso } \\
\text { F, Silva ICM, Granjeiro R, } \\
\text { Carmo TG. }\end{array}$ & Iatrogenia no cuidado da enfermagem: implicações éticas e penais. & 2009 \\
\hline $\begin{array}{l}\text { Figueiredo BO, De Paula LB, } \\
\text { Hipólito RL. }\end{array}$ & Ocorrência de iatrogenia pela enfermagem na unidade de terapia intensiva. & 2009 \\
\hline Martins JT, Robazzi MLCC. & $\begin{array}{l}\text { O trabalho do enfermeiro em unidade de terapia intensiva: sentimentos de } \\
\text { sofrimento. }\end{array}$ & 2009 \\
\hline Santos JC, Ceolim MF. & latrogenias de enfermagem em pacientes idosos hospitalizados. & 2009 \\
\hline $\begin{array}{l}\text { Campos GF, Sena ACM, } \\
\text { Fernandes DJM, Cruz TF, } \\
\text { Silva EAC. }\end{array}$ & Cuidados de enfermagem e ocorrências iatrogênicas na UTI. & 2008 \\
\hline Apolinário RS, Corrêa AK. & $\begin{array}{l}\text { Educação profissional: vivencia do educando de enfermagem no cuidado ao } \\
\text { doente crítico. }\end{array}$ & 2007 \\
\hline Toffoletto MC, Padilha KG. & $\begin{array}{l}\text { Consequências dos erros de medicação em unidades de terapia intensiva e } \\
\text { semi-intensiva. }\end{array}$ & 2006 \\
\hline Vargas D, Braga AL. & O enfermeiro da unidade de tratamento intensivo: refletindo sobre seu papel. & 2006 \\
\hline Silva AEBC, Cassiani SHB. & $\begin{array}{l}\text { Erros de medicação em hospital universitário: tipo, causas, sugestões e } \\
\text { providências. }\end{array}$ & 2004 \\
\hline Camargo MNV, Padilha KG. & Ocorrências iatrogênicas com medicação em unidades de terapia intensiva. & 2003 \\
\hline Decesaro MN, Padilha KG. & $\begin{array}{l}\text { latrogenia na assistência de enfermagem durante internação em UTI: queda de } \\
\text { pacientes. }\end{array}$ & 2002 \\
\hline Guedes EP, Marra CC. & Prevenção de iatrogenias em idosos em unidade de terapia intensiva. & 2002 \\
\hline $\begin{array}{l}\text { Padilha KG, Kitahara PH, } \\
\text { Gonçalves CCS, Sanches ALC. }\end{array}$ & $\begin{array}{l}\text { Ocorrências iatrogênicas com medicação em unidades de terapia intensiva: } \\
\text { condutas adotadas e sentimentos expressos pelos enfermeiros. }\end{array}$ & 2002 \\
\hline Madalosso ARM. & $\begin{array}{l}\text { latrogenia do cuidado de enfermagem: dialogando com o perigo no quotidiano } \\
\text { profissional. }\end{array}$ & 2000 \\
\hline Moreira RS, Padilha KG. & $\begin{array}{l}\text { Ocorrências iatrogênicas com pacientes submetidos à ventilação mecânica em } \\
\text { unidade de terapia intensiva. }\end{array}$ & 2001 \\
\hline Silva SC, Padilha KG. & $\begin{array}{l}\text { Parada cardiorrespiratória na unidade de terapia intensiva: considerações teóricas } \\
\text { sobre os fatores relacionados às ocorrências iatrogênicas. }\end{array}$ & 2001 \\
\hline
\end{tabular}

Quanto à indexação das revistas em que foram publicados estes artigos observamos a predominância com $9(45,0 \%)$ foram publicados em revistas com indexação internacional $\left(A_{2}\right)$, enquanto $5(25,0 \%)$ publicados em revistas de publicação nacional $\left(B_{1}, B_{3}, B_{4}\right.$ e $\left.B_{5}\right)$ e 6 $(30,0 \%)$ em revistas ainda não indexadas. Vale ressaltar que todas as revistas indexadas ou não, exigem rigor científico das pesquisas para serem publicadas.

Conforme mostra a tabela 5 foram selecionados 20 publicação. artigos para integrar a amostra deste trabalho, mostrando assim seus autores, títulos e ano de 
latrogenias: ações do enfermeiro na prevenção de ocorrências iatrogênicas em unidade de terapia intensiva

\section{Principais Ocorrências I atrogênicas na UTI}

As iatrogenias relacionadas aos cuidados de enfermagem, percebe-se a frequência das ocorrências iatrogênicas no contexto da enfermagem, estas, estão presentes em situações corriqueiras vivenciadas diariamente no ambiente de trabalho deste profissional onde a dinâmica do ambiente exige uma manipulação frequente dos pacientes que são totalmente dependentes da assistência de enfermagem ${ }^{8}$.

As principais ocorrências encontradas na literatura pesquisada no cotidiano da enfermagem na unidade de terapia intensiva são: a falta de interesse pelas atividades; a preocupação somente em gerenciar a equipe, não se preocupando em manter o contato com o paciente; falta de motivação e falta de informação; a necessidade frequente de substituir outros profissionais da equipe nas suas tarefas; a não realização da sistematização e organização dos documentos sobre o cuidado de enfermagem; a falta de supervisão adequada sobre o trabalho de técnicos e auxiliares de enfermagem; a realização incorreta dos procedimentos aprendidos durante a vida acadêmica; pouco interesse sobre educação continuada para si mesmo e sua equipe; carga horária extensa; o não cumprimento das leis que regem o exercício profissional e pouca autoestima com a vida profissional.

Em estudo realizado por outros pesquisadores, mostrou que as ocorrências iatrogênicas decorrentes por administração de medicamentos, os resultados obtidos na pesquisa revelaram uma realidade difícil e preocupante. Os dados mostraram que as situações mais frequentes que podem desencadear as ocorrências foram: administração incorreta dos medicamentos $(35,1 \%)$; falha na prescrição $(29,3 \%)$ e falha no preparo da medicação $(19,1 \%)$. Apesar da gravidade dos dados, além de indicar a existência do problema, é importante que sua discussão abra caminhos para a compreensão da complexidade do assunto ${ }^{9}$.

Os problemas inerentes à prática assistencial, objetivamente as consequências dos erros de medicação nas condições clínicas do paciente grave e na carga de trabalho da equipe de enfermagem com o uso de instrumentos de medida objetivos, validados e reconhecidos internacionalmente, o que se tornou possível, no entanto, com o desenvolvimento dos diferentes índices atualmente disponíveis ${ }^{10}$.

\section{Ações do Enfermeiro na Prevenção das I atrogenias}

Ao enfermeiro de terapia intensiva compete cuidar da pessoa nas diferentes situações críticas dentro da unidade de terapia intensiva, de forma integrada e contínua com os membros da equipe de saúde, para isso o enfermeiro de unidade de terapia intensiva precisa pensar criticamente analisando os problemas e encontrando soluções para os mesmos, assegurando sempre sua prática dentro dos princípios éticos e bioéticos da profissão ${ }^{11}$.

O principal método para minimizar a falha humana no desencadeamento de ocorrências iatrogênicas é simplificar os processos, criar protocolos de administração de medicamentos voltados à necessidade do atendimento, desde a prescrição médica eletrônica; sem abreviações; com horários padronizados; distribuição de medicamentos da farmácia sempre supervisionada pelo farmacêutico e preferencialmente em dose unitária ${ }^{12}$.

O enfermeiro da UTI precisa pensar criticamente analisando os problemas e encontrar soluções para os mesmos, assegurar sempre a prática dentro dos princípios éticos da profissão. Respaldar no conhecimento científico a sistematização e decisão sobre o uso de recursos humanos, físicos, materiais e de informação apropriados visando o trabalho em equipe para que possa conduzir o atendimento do paciente com segurança ${ }^{13}$.

O enfermeiro tem que estar preparado para cuidar dos pacientes na unidade de terapia intensiva, pois estes pacientes não desejam mais serem submetidos simplesmente à cura, querem ser tratado como gente, partilhar e interagir nos cuidados para alcançar um bem viver. Não aceitam mais o tão perigoso fazer para produzir, que empobrece o viver do ser humano, que não considera o preço e os sacrifícios que são impostos para serem alcançados ${ }^{14}$.

$\mathrm{Na}$ tabela 6 buscamos destacar em cada artigo selecionado as informações sobre as medidas preventivas, esse processo nos levou a identificar que diferentes autores destacam medidas iguais ou semelhantes para prevenção das iatrogenias. 
latrogenias: ações do enfermeiro na prevenção de ocorrências iatrogênicas em unidade de terapia intensiva

Tabela 6. Informações expressas nos textos segundo as ações do enfermeiro na prevenção das iatrogenias. São Paulo, 2012

Referência Bibliográfica
1.0 papel do enfermeiro na
prevenção de ocorrências
iatrogênicas em unidade de
terapia intensiva.
2.latrogenias na assistência de
enfermagem: características da
produção científica no período
de 2000 a 2009 .

\section{Autor I Ano}

Maia LFS,

Alves FG.

2011

\section{Farias GM}

Costa IKF,

Rocha KMM,

Fritas MCS,

Dantas RAN.

2010

\begin{tabular}{lrl}
\hline 3.latrogenias nas unidades de & Vargas MAO, \\
terapia intensiva: & Ramos FRS. \\
dramaticidade dos problemas & 2010
\end{tabular}

bio/éticos contemporâneos.

4. Ocorrências iatrogênicas nas unidades de terapia intensiva: enfoque nas ações da equipe de enfermagem.

\section{Ribeiro}

MPFN,

Teodoro

OS, Brasileiro

ME. 2010

5.latrogenia no cuidado da Cortez EA, enfermagem: implicações Marçal C,

éticas e penais.

Cardoso $\mathrm{F}$

Silva ICM,

Granjeiro R,

Carmo TG.

2009

6.Ocorrência de iatrogenia pela Figueiredo

enfermagem na unidade de BO, De Paula

terapia intensiva.

LB, Hipólito

RL. 2009

7.0 trabalho do enfermeiro em unidade de terapia intensiva: sentimentos de sofrimento.

Martins JT,

Robazzi

MLCC. 2009
MWR, Borges

Utilização de métodos que otimizem e tornem eficiente a educação continuada deve ser uma prioridade nas instituições de saúde, já que é através dela que seus profissionais se tornam incentivados $\mathrm{e}$ atualizados com as novas técnicas, procedimentos e equipamentos que surgem a cada momento.

Trabalho em conjunto com os administradores, diretoria e profissionais de enfermagem das instituições hospitalares. Condições de trabalho da equipe, no que se refere à quantidade e qualidade dos profissionais, assim como do material técnico e programas de educação permanente, remuneração condizente com sua ocupação, autonomia status profissional, interação, respeito entre outros requisitos necessários à satisfação no trabalho.

Agregar e transmitir conhecimento, operar mesmo na formação do indivíduo como sujeito. É, pois, uma reforma da atitude, das ações do sujeito que, também, está incluído no complexo processo de prevenção das iatrogenias, dos eventos adversos. Reflexão sobre o agir do enfermeiro em um contexto permeado pela possibilidade, sempre latente, possibilidade de corrigir falhas, não tanto ou não apenas no conhecimento, não tanto ou não apenas na lei, mas na prática de si mesmo.

Cursos de qualificação periodicamente para que possa atuar com segurança e, assim,
prevenir tais ocorrências. Treinamento contínuo e atualização dos conhecimentos e técnicas que permeiam a assistência.

A necessidade de reafirmar a questão educativa como compromisso para o crescimento profissional e pessoal, visando a melhorar a qualidade da prática profissional. A formação profissional de qualidade deve ter sólida base de formação geral, que não completa na escola, mas sim dentro do processo evolutivo do ser humano.

Para que haja a prevenção das ocorrências iatrogênicas, principalmente na UTI, é preciso que o enfermeiro responsável pelo setor tenha consciência que a iatrogenia é presente, reconhecer os pontos em que ele e sua equipe estão falhando na assistência e elaborar planos para diminuir esses erros, além de intensificar o trabalho de educação continuada. Isso para que os pacientes internados e que necessitam dos seus cuidados e de sua equipe estejam longe de riscos causados pela própria Enfermagem.

Despertar nos trabalhadores maior reconhecimento dos seus sentimentos e da própria vida existencial no mundo e, para isso, é preciso criar novas habilidades, nova consciência, ou seja, novos seres capazes de ser ao mesmo tempo de ter. Assim, poderão cuidar melhor da sua vida psíquica e física. Sendo isso um ato de responsabilidade direta do próprio ser humano e indiretamente das pessoas e instituições que os rodeiam.

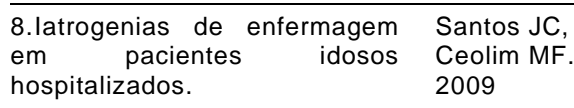

A criação de uma enfermaria voltada para o público idoso, para oferecer-lhe cuidados específicos, o que não acontece atualmente já que as enfermarias estudadas atendem adultos em geral. Literatura com estudos que focalizem objetivamente as ocorrências iatrogênicas aqui encontradas e as suas consequências para o paciente idoso em especial, e que abordem as ocorrências iatrogênicas em outras unidades além daquelas de cuidados intensivos, das quais se ocupam a maior parte dos estudos.

9. Cuidados de enfermagem e Campos GF, ocorrências iatrogênicas na Sena ACM.

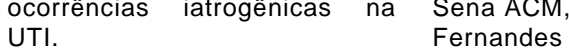
continuada com os seus colaboradores que elucidem o que e iatrogenia discutindo os DJM, Cruz TF, cenários para entender as causas do problema e propor melhoria, a fim de transformar

Silva EAC. o paciente que estiver sob responsabilidade de sua equipe. 2008

10. Educação profissional:
vivencia do educando de Corrêa AK. vivencia do educando de Corrêa AK. enfermagem no cuidado ao 2007 doente crítico.

A formação do profissional de saúde é predominantemente técnica e isto leva a repensar a forma como o cuidado do outro está sendo aprendido pelo aluno. Ao invés de instruir o educando com uma falsa cultura, com saberes pré-definidos e orientados para o aprendizado de determinadas habilidades, é preciso sem isolá-los da sociedade em que vivem. Sem dúvida, as pessoas podem ser treinadas, mas só o ser humano é capaz de ensinar e aprender, atividades essencialmente humanas e profundamente significativas para sua existência.

11. Consequências dos erros de medicação em unidades de terapia intensiva e semi- 2006 intensiva.
Toffoletto MC,

Padilha KG.

2006
Implantação de sistemas de registros de ocorrências, inclusive por meio de programas oficiais de gerenciamento de riscos nas instituições hospitalares, a realidade mostra a necessidade de engajamentos mais efetivos. 
latrogenias: ações do enfermeiro na prevenção de ocorrências iatrogênicas em unidade de terapia intensiva

\begin{tabular}{lll}
\hline 12. O enfermeiro da unidade & Vargas $D$, \\
de tratamento intensivo: & $\begin{array}{l}\text { Braga AL. } \\
\text { refletindo sobre seu papel. }\end{array}$ & 2006
\end{tabular}

\begin{tabular}{lcrl}
\hline 13. Erros de medicação em & Silva AEBC, \\
hospital & universitário: & tipo, & Cassiani SHB. \\
causas, & sugestões & e & 2004
\end{tabular}

providências.

14. Ocorrências iatrogênicas Camargo com medicação em unidades MNV, Padilha de terapia intensiva.
Compromisso contínuo com seu próprio desenvolvimento profissional, capaz de atuar nos processos educativos dos profissionais da equipe de saúde, em situações de trabalho, proporcionando condições para que haja benefício mútuo entre os profissionais, responsabilizando-se ainda pelo processo de educação em saúde dos indivíduos e familiares sob seu cuidado, reconhecendo o contexto de vida e os hábitos socioeconômico e cultural, contribuindo com a qualificação da prática profissional, construindo novos hábitos e desmistificando os conceitos inadequados atribuídos a UTI.

Implantar medidas como treinamento sobre os erros de medicação, adoção de relatórios de ocorrência e erros sem consequentes punições aos envolvidos, implementado medidas administrativas voltadas ao planejamento do sistema de medicação e não as pessoas, sendo estratégias iniciais em busca de maior segurança para o paciente.

Garantir um espaço físico adequado e momento definido para o preparo da medicação, sem constantes interrupções, medidas simples e eficazes no controle de risco das ocorrências iatrogênicas. Busca pela qualidade na administração de medicamentos, valorização da atividade por parte dos profissionais da equipe de enfermagem, enfatizando a necessidade de formação acadêmica adequada para realizá-la com segurança.

\begin{tabular}{|c|c|}
\hline $\begin{array}{l}\text { 15. Iatrogenia na assistência } \\
\text { de enfermagem } \\
\text { internação em UTI: queda de } \\
\text { pacientes. }\end{array}$ & $\begin{array}{l}\text { Decesaro MN, } \\
\text { Padilha KG. } \\
2002\end{array}$ \\
\hline
\end{tabular}

Adoção de medidas preventivas para tais eventos, tanto no que se refere à capacitação e treinamento dos profissionais como na adequação da infraestrutura da unidade, com vistas a uma assistência de enfermagem com qualidade. Indicam ainda que não basta a melhoria de segmentos isolados na busca de qualidade, mas é preciso somar esforços para o alcance de um objetivo comum, ou seja, a melhoria da assistência, com a aplicação de um cuidado mais individualizado e holístico.

\begin{tabular}{ll}
\hline $\begin{array}{l}\text { 16. Prevenção de iatrogenias } \\
\text { em idosos em unidade de } \\
\text { terapia intensiva }\end{array}$ & $\begin{array}{l}\text { Guedes EP, } \\
\text { Marra CC. } \\
2002\end{array}$ \\
\hline $\begin{array}{l}\text { 17. Ocorrências iatrogênicas } \\
\text { com medicação em unidades } \\
\text { de terapia intensiva: }\end{array}$ & $\begin{array}{l}\text { Kitahara PH, } \\
\text { Gonçalves }\end{array}$ \\
condutas adotadas e & CCS, \\
sentimentos expressos pelos & Sanches \\
enfermeiros. & ALC. 2002 \\
\hline $\begin{array}{l}\text { 18. latrogenia do cuidado de } \\
\text { enfermagem: dialogando com }\end{array}$ & Madalosso \\
o perigo no quotidiano 2000 \\
profissional.
\end{tabular}

Assegurar a continuidade da assistência, promover a qualidade da assistência de enfermagem, minimizando as ocorrências iatrogênicas que podem ser diminuídas através de uma assistência específica e não generalizada para cada cliente geriátrico, promover nas UTIs um processo de educação continuada sistematizada.

Incentivo para a notificação dos erros, a monitorização das ocorrências e fatores a elas relacionados, bem como ações menos punitivas e mais educativas venham favorecer a diminuição dos erros que tantos malefícios causam aos pacientes e a todos os envolvidos na sua assistência, como ficou demonstrado nesta investigação.

A enfermagem tem que estar preparada para cuidar dos indivíduos deste novo tempo, que não desejam mais serem submetidos simplesmente à cura, querem ser tratados como gente, partilhar e interagir nos cuidados para alcançar um bem viver. Não aceitam mais o tão perigoso fazer para produzir, que empobrece o viver do ser humano, que não considera o preço e os sacrifícios que são impostos para serem alcançados.

19. Ocorrências iatrogênicas Moreira RS, com pacientes submetidos à Padilha KG. ventilação mecânica em 2001 unidade de terapia intensiva.

A implementação de medidas preventivas de ocorrências iatrogênicas, muito mais do que proteger apenas o paciente de consequências indesejáveis, beneficia tambem profissionais, familiares, instituição e sociedade, razão mais do que para ser encarada como meta a ser alcançada por todos. 20. Parada cardiorrespiratória Silva SC, Implementação de medidas preventivas que enfoquem as ocorrências iatrogênicas na unidade de terapia Padilha KG intensiva: considerações teóricas sobre os fatores relacionados às ocorrências no atendimento à PCR dentro de um contexto sistêmico, de forma a identificar e intervir nos pontos vulneráveis de qualquer um dos elementos constitutivos do cuidado sejam os recursos humanos, recursos materiais e equipamentos, administrativos e técnicos.

iatrogênicas.

Fonte: Biblioteca Virtual e Física, 2011.

As informações disponíveis no texto segundo os objetivos da pesquisa resultaram no melhor entendimento do tema e mostrou o quanto o profissional enfermeiro deve estar presente e fazendo melhorias para prevenção das iatrogenias.

A atuação do enfermeiro em unidade de terapia intensiva visa ao atendimento do cliente, incluindo-se o diagnóstico de sua situação, intervenções e avaliação dos cuidados específicos de enfermagem, a partir de uma perspectiva humanista voltada para a qualidade qualidade de vida. Considerando que um dos indicadores dessa qualidade é a higidez do cliente a qual conduz ao seu bem estar nas dimensões física, mental e espiritual, acredita-se que a atuação de enfermagem pode ser favorecida pela institucionalização de um instrumento de avaliação de enfermagem que oriente os profissionais para, por exemplo, predizer se o cliente admitido na UTI apresenta ou não, fatores de risco.

Neste sentido para que haja melhor qualidade do cuidado de enfermagem é necessária uma formação de 
latrogenias: ações do enfermeiro na prevenção de ocorrências iatrogênicas em unidade de terapia intensiva

qualidade voltada aos acadêmicos de enfermagem de modo que estes se apropriem de conhecimentos científicos pertinentes a essa temática e se conscientizem da sua importância para uma assistência de enfermagem com boa qualidade.

\section{Considerações Finais}

Ressaltamos a necessidade de ampliação no desenvolvimento da pesquisa sobre as iatrogenias que na prática assistencial, as intervenções preventivas e terapêuticas, ainda se encontram em fase de conhecimento dos enfermeiros, tornando-se umas práticas não institucionalizadas.

Salientamos que o enfermeiro e todos os profissionais envolvidos devem reconhecer as limitações envolvidas e buscar superá-las, proporcionando avanços e ampliando os estudos clínicos que possam subsidiar a prática profissional.

É fundamental a adoção de protocolos assistenciais que contemple a magnitude desses fatores e condições identificadas e discutidas, com vista a melhorar a qualidade da assistência, tornando-a mais humanizada, reduzindo complicações decorrentes das iatrogenias.

\section{Conclusão}

Após a análise dos artigos e com base no levantamento teórico feito acerca das ocorrências iatrogênicas em UTI, salientamos ainda que não basta a melhoria de segmentos isolados na busca de qualidade, mas é preciso somar esforços para o alcance de um objetivo comum, ou seja, a melhoria da assistência, com a aplicação de um cuidado mais individualizado e holístico.

Ao avaliar os artigos científicos pesquisados relacionados com as iatrogenias, observamos nos trabalhos a grande importância do enfermeiro conhecer os fatores de risco e colocá-los em prática com medidas preventivas.

Faz-se necessária maior socialização do conhecimento na prevenção das iatrogenias a partir das evidências e pesquisas científicas para desenvolvimento de políticas com vistas à institucionalização de métodos preventivos.
Ficou evidente em nosso estudo que as ações do enfermeiro para a prevenção das iatrogenias devem ser prolongadas, os métodos preventivos privilegiados, visto que inversamente prevenir é melhor que remediar.

Concluímos que processo de prevenção das ocorrências iatrogênicas na UTI requer esforço contínuo de todos dos membros da equipe que atuam no setor e não apenas do enfermeiro, porque todos tem a responsabilidade de lidar com o paciente, sobretudo, lutar diariamente contra a mecanização de seu trabalho.

\section{Referências}

1. Maia LFS, Nascimento EB, Gerardini V. O avanço tecnológico e o cuidado humanizado em centro cirúrgico. São Paulo: Revista SOBECC. 2006; 11:26-31.

2. Maia LFS, Alves FG. O papel do enfermeiro na prevenção de ocorrências iatrogênicas em unidade de terapia intensiva. São Paulo: Revista Recien. 2011; 1(3): 19-24.

3. Maia LFS. Humanização em unidade de terapia intensiva: a enfermagem e o cuidado humanizado. São Paulo: Recien. 2010; 1:06-11.

4. Araújo $A D$. Trabalho no centro de terapia intensiva: perspectivas da equipe de enfermagem. Revista Mineira de Enfermagem. 2005; 9(1):20-28.

5. Nogueira LA, Felipes L, Coimbra JAH. Reflexões sobre a problemática iatrogenia e o código de ética. Seminário Nacional Estado e Políticas Sociais no Brasil. 2005.

6. Padilha, KG. A prática de enfermagem em UTI e as ocorrências iatrogênicas: considerações sobre o contexto atual. Rev. Paul. Enferm. 2000; 19(3): 49-56.

7. GIL, A. C. Métodos e técnicas de pesquisa social. São Paulo: Atlas, 2000.

8. Campos GF, Sena ACM, Fernandes DJM, Cruz TF, Silva EAC. Cuidados de enfermagem e ocorrências iatrogênicas na UTI. 2008. Disponível em: <http://webartigos.com>. Acesso em: 27 Nov 2011.

9. Camargo MNV, Padilha KG. Ocorrências iatrogênicas com medicação em unidades de terapia intensiva. Acta Paul Enf. 2003; 16(4)

10. Toffoletto MC, Padilha KG. Consequências dos erros de medicação em unidades de terapia intensiva e semiintensiva. Rev Esc Enferm USP. 2006; 40(2):247-52. 
11. Vargas D, Braga AL. O enfermeiro de unidade de tratamento intensivo: refletindo sobre seu papel. Ribeirão Preto: Rev. Latino Am. Enfermagem. 2002; 10(2).

12. Ribeiro MPFN, Teodoro MWR, Borges OS, Brasileiro ME. Ocorrências iatrogênicas nas unidades de terapia intensiva: enfoque nas ações da equipe de enfermagem. Revista Eletrônica de Enfermagem do Centro de Estudos de Enfermagem e Nutrição. 2010; 1(1): 1-16. Disponível em: <http://www.ceen.com.br>. Acesso em: 15 Dez 2011.
13. Santos GA, Ferrari D. Atuação do enfermeiro intensivista no programa saúde da família: relato de experiência. Revista Intensiva. 2010; 4(26):18-22.

14. Madalosso ARM. Iatrogenia do cuidado de enfermagem: dialogando com o perigo no quotidiano profissional. Ribeirão Preto: Rev. Latino Am Enfermagem. 2000; 8(3):11-17. 